\title{
Bilateral Tactile Feedback-Enabled Training for Stroke Survivors Using Microsoft Kinect ${ }^{\mathrm{TM}}$
}

\author{
Abbas Orand ${ }^{1}$ (D), Eren Erdal Aksoy ${ }^{1}$, Hiroyuki Miyasaka ${ }^{2}{ }^{(D}$, Carolyn Weeks Levy $^{3}$, Xin Zhang $^{3}$ \\ and Carlo Menon ${ }^{3, *(D)}$ \\ 1 Department of Intelligent Systems and Digital Design, School of Information Technology, \\ Halmstad University, Spetsvinkelgatan 29, 30250 Halmstad, Sweden \\ 2 Department of Rehabilitation, Fujita Health University Nanakuri Memorial Hospital, 424-1 Oodori-cho, Tsu, \\ Mie 514-1296, Japan \\ 3 Schools of Mechatronics Systems Engineering and Engineering Science, Simon Fraser University, \\ 250-13450 102 Avenue, Surrey, BC V3T 0A3, Canada \\ * Correspondence: cmenon@sfu.ca; Tel.: +(001)-778-782-6860
}

Received: 13 June 2019; Accepted: 5 August 2019; Published: 8 August 2019

\begin{abstract}
Rehabilitation and mobility training of post-stroke patients is crucial for their functional recovery. While traditional methods can still help patients, new rehabilitation and mobility training methods are necessary to facilitate better recovery at lower costs. In this work, our objective was to design and develop a rehabilitation training system targeting the functional recovery of post-stroke users with high efficiency. To accomplish this goal, we applied a bilateral training method, which proved to be effective in enhancing motor recovery using tactile feedback for the training. One participant with hemiparesis underwent six weeks of training. Two protocols, "contralateral arm matching" and "both arms moving together", were carried out by the participant. Each of the protocols consisted of "shoulder abduction" and "shoulder flexion" at angles close to 30 and 60 degrees. The participant carried out 15 repetitions at each angle for each task. For example, in the "contralateral arm matching" protocol, the unaffected arm of the participant was set to an angle close to 30 degrees. He was then requested to keep the unaffected arm at the specified angle while trying to match the position with the affected arm. Whenever the two arms matched, a vibration was given on both brachialis muscles. For the "both arms moving together" protocol, the two arms were first set approximately to an angle of either 30 or 60 degrees. The participant was asked to return both arms to a relaxed position before moving both arms back to the remembered specified angle. The arm that was slower in moving to the specified angle received a vibration. We performed clinical assessments before, midway through, and after the training period using a Fugl-Meyer assessment (FMA), a Wolf motor function test (WMFT), and a proprioceptive assessment. For the assessments, two ipsilateral and contralateral arm matching tasks, each consisting of three movements (shoulder abduction, shoulder flexion, and elbow flexion), were used. Movements were performed at two angles, 30 and 60 degrees. For both tasks, the same procedure was used. For example, in the case of the ipsilateral arm matching task, an experimenter positioned the affected arm of the participant at 30 degrees of shoulder abduction. The participant was requested to keep the arm in that position for $\sim 5 \mathrm{~s}$ before returning to a relaxed initial position. Then, after another $\sim 5$-s delay, the participant moved the affected arm back to the remembered position. An experimenter measured this shoulder abduction angle manually using a goniometer. The same procedure was repeated for the 60 degree angle and for the other two movements. We applied a low-cost Kinect to extract the participant's body joint position data. Tactile feedback was given based on the arm position detected by the Kinect sensor. By using a Kinect sensor, we demonstrated the feasibility of the system for the training of a post-stroke user. The proposed system can further be employed for self-training of patients at home. The results of the FMA, WMFT, and goniometer angle measurements showed improvements in several tasks, suggesting a positive effect of the training system and its feasibility for further application for stroke survivors' rehabilitation.
\end{abstract}


Keywords: Kinect; stroke rehabilitation; bilateral training; tactile feedback

\section{Introduction}

A stroke is caused by interruptions in the blood supply to the brain [1]. It is serious and disabling [2] and is the cause of adult disability in many countries [2,3]. The hemiparesis of the contralateral upper limb is the most common deficit after stroke and affects more than $80 \%$ of stroke survivors [4]. Upper extremity (UE) impairments affect the activities of daily living of stroke survivors by causing disability in common activities such as reaching $[5,6]$. Meaningful hand activity six months post-stroke can be minimal [6], and long-term health effects continue to affect the quality of life of the patient. Therefore, improved interventions for the rehabilitation of stroke survivors are essential. However, traditional rehabilitation interventions result in continued impairment of stroke survivors [7,8]. In order to improve rehabilitation protocols and results, new approaches such as bilateral training, feedback application, and low-cost 3D motion capture systems such as Kinect ${ }^{\mathrm{TM}}$ (Kinect is manufactured by Microsoft and is a motion sensing device) were investigated in this study.

\subsection{Bilateral Training}

Studies have shown that unaffected and affected limbs that were simultaneously moved in a bilateral training protocol were effective in the recovery of motor function in hemiplegic patients [9-12]. Activation of the nervous system and stimulation of the cerebral hemisphere by bimanual training were shown in another study [13]. Another study showed that contralateral movement was controlled by $90 \%$ of the nerve fibers in the cerebral cortex and the spinal cord, while the remaining 10\% controlled ipsilateral movement [14]. Activities of daily living (ADLs) also require the use of both hands, making bilateral rehabilitation training necessary [15,16]. In a structured review and meta-analysis of studies made on bilateral movement training and stroke recovery, Cauraugh et al. [17] identified 48 potential research studies, of which 25 were compared to determine the cumulative effect of bilateral arm training on motor capabilities post-stroke. They concluded that pure bilateral or active and/or passive movement training protocols do not contribute to significant motor recovery. The applications of bimanual training with robots [18-20] and rhythmic auditory cueing [21] were investigated in the rehabilitation of stroke survivors. The possibility of the concept of bimanual training and companied devices in extending contemporary therapies is accepted. The combination of bimanual training with rhythmic auditory cueing was found to be partially efficient in the rehabilitation of chronic stroke patients.

\subsection{Tactile Feedback}

Afferent nerves carry tactile messages such as vibration, pressure, and stretch to the brain [22,23]. Shull et al. [24] defined haptics as the sense of touch, which includes vibration. They classified vibration feedback in the partial impairment category, which requires sensory augmentation. Haptic wearables were shown to be useful for partial impairment by facilitating motor control and rehabilitation [25], and a haptic wearables application was even beneficial for individuals with intact sensory information for behavioral deficit correction [24]. In a study conducted by Stepp and Matsuoka [26] on the differences between stimulation sites during vibrotactile feedback, they found that the location of skin stimulation was of less importance than other factors, such as learning rates. Vibrotactile feedback was applied for proprioception feedback [27] and in stroke subjects [28]. The sensory feedback was found to be directly correlated with accuracy and to be beneficial for increasing subject confidence [28]. Held et al. [28] found vibrotactile feedback to be more acceptable to stroke patients. They concluded that it can be used as a telerehabilitation device for training. 


\subsection{Kinect Application}

Microsoft's Kinect is a 3D motion capture system [29]. It can identify 25 body joint centers at a frequency of $30 \mathrm{~Hz}$ using a personal computer [30]. Considering Kinect's great properties, such as portability, low cost, and its markerless design, it can easily be used for measuring human movement [30]. The validity of Kinect in a clinical study of people with Parkinson's disease yielded a good interclass correlation coefficient, $>0.9$, and a Pearson's $r>0.9$ [31]. Webster et al. [32] carried out a systematic review of Kinect applications in the elderly for stroke rehabilitation. They concluded that the Kinect has great potential in providing therapy for such individuals. The Kinect was also applied for building financially accessible and medically beneficial systems for patient use [32]. The Kinect has been used for a virtual reality rehabilitation system [33], a virtual exercise guide and game-based rehabilitation system [34-36], an interactive visuotactile 3D virtual environment [37], and a brain-controlled interface-driven robotic upper limb exoskeleton [38].

\subsection{Proprioception Training}

Proprioception is the sense of body position [39]. It is the ability of the central nervous system to determine body part positioning in time [40] and space [41]. Proprioception is important for sensorimotor control and is required for muscle stiffness regulation, joint stability and speed, coordination, and balance [39,41-43]. Therefore, intact proprioception is essential for movement control and functioning [44]. Proprioception training has been shown to decrease the incidence of injuries and to improve proprioception accuracy in trained healthy subjects compared to nontrained healthy subjects [45-48]. A proprioception deficit is common among stroke patients. A recent research study [41] reported that up to $85 \%$ of stroke patients' proprioception was affected after stroke. For those post-stroke patients with affected UE, the proprioception deficit was reported to be $30 \%$ to $48 \%$ [43]. Therefore, interventions for proprioceptive rehabilitation are crucial for stroke patients suffering from a proprioception deficit.

A self-training/rehabilitation system for stroke survivors would be beneficial for society. The burden of rehabilitation costs on the healthcare system has a great impact on the quality of care that stroke survivors receive [49]. Large numbers of in- and outpatients compared to low numbers of therapists and caregivers have significant health-related consequences for caregivers as well as patients [50]. Self-training at home is one option that could provide relief to the healthcare system and reduce the burden on therapists and clinicians. In this study, our objective was to actively involve the user through a bimanual training protocol combined with tactile feedback to improve his motor learning and neuromotor rehabilitation. We added the Kinect to investigate its potential for a self-training/rehabilitation system and applied its joint position data for providing the participant with tactile feedback. Our goal was to develop an affordable and medically beneficial system. We hypothesized that the combined bimanual movement with tactile feedback would result in improved rehabilitation output.

\section{Methods}

A 65-year-old male left chronic stroke survivor with a stroke onset of 8 years was recruited for the study. He did not receive any rehabilitation treatment during the study, which took place at Simon Fraser University (SFU). He did not have any spasticity in his affected left limb. The Office of Research Ethics at SFU approved the clinical study protocol (No. 2012s0711), and the inclusion criteria were as follows:

- the absence of primary joint trauma and dislocation (elbow and shoulder);

- no history of fractures and peripheral nerve paralysis;

- normal cognitive score $>25$ with the Mini-Mental State Examination [51];

- ability to understand instructions in English;

- ability to sit on a chair and to perform tasks; 
- $\quad$ mild to moderate paralysis (Fugl-Meyer assessment (FMA) > 19);

- more than 60 degrees of active range of motion for shoulder abduction, shoulder flexion, and elbow flexion.

\subsection{Training Protocol}

The participant came to the lab 3 times per week for 6 weeks. Two training protocols of "contralateral arm matching" and "both arms moving together" were used during the training sessions. Each of these training sessions lasted a maximum of $1 \mathrm{~h}$. Two arm bands, each connected to a vibrating motor, were placed on the participant's brachialis muscles. The two motors were connected to a receiver. The participant sat in an armchair in a comfortable position and was blindfolded (Figure 1) in order to eliminate the influence of vision on proprioception. For both training protocols, the participant carried out two tasks of "shoulder abduction" and "shoulder flexion" at angles close to 30 and 60 degrees. The two angles were both within the range of motion of the participant. The participant carried out 15 repetitions for each angle in two sets. The first set consisted of 10 repetitions and the second set included 5 repetitions for a total of 15 repetitions. The participant was allowed a short break between sets.

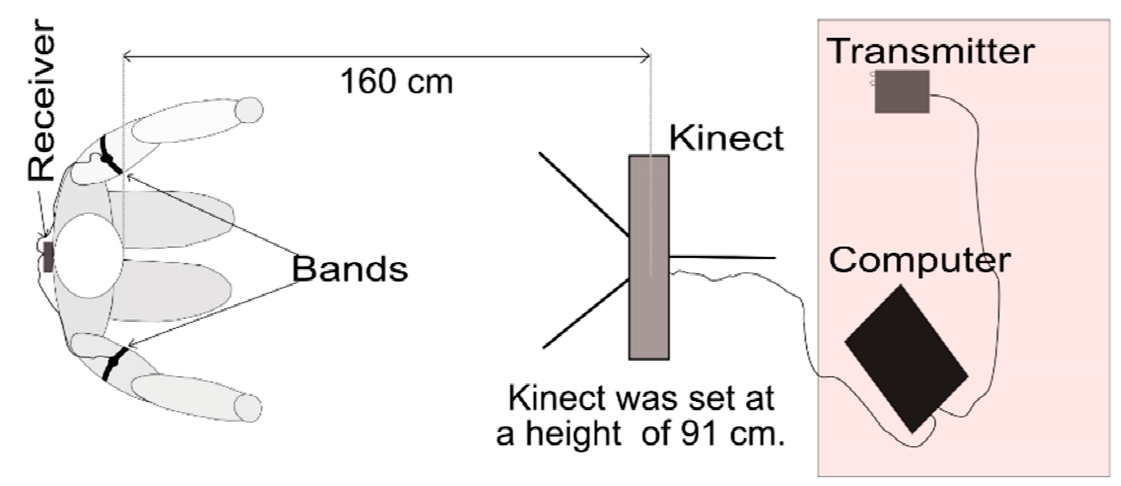

A

B

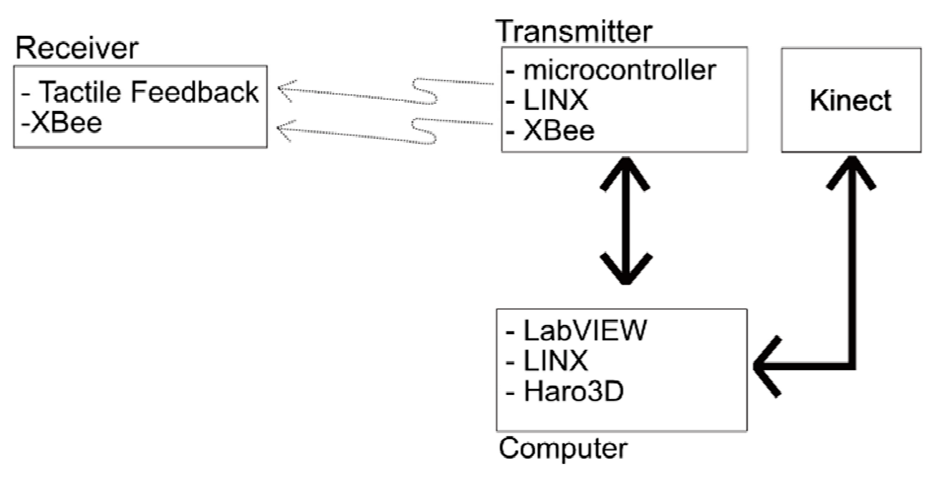

Figure 1. The training/rehabilitation system is shown: (A) It consists of a receiver with two bands that are put on the shoulders of the user for tactile feedback; a Kinect sensor placed $160 \mathrm{~cm}$ away from the user at a height of $91 \mathrm{~cm}$; a transmitter that communicates with the receiver wirelessly, sending commands for tactile feedback, with two light emitting diodes (LEDs) serving as left- and right-arm visual feedback; and a computer connected to the Kinect and transmitter, which processes the joint information and executes a command according to the algorithm. (B) Hardware and software applied in the training system.

For example, in the "contralateral arm matching" protocol, the unaffected arm of the participant was set to an angle close to 30 degrees. Then, he was requested to keep the unaffected arm in the specified angle while trying to match the position with the affected arm. Whenever the two arms 
matched, a vibration was delivered to both brachialis muscles. In the "both arms moving together" protocol, the two arms were first approximately set to an angle of either 30 or 60 degrees. Then, the participant had to return to a relaxed position before moving both arms back to the remembered specified angle. Whichever arm lagged behind the other arm in resuming the specified angle received a vibration. Therefore, the participant's goal was to move both arms to the specified angle without any lag, consequently not getting any vibration in his arms.

\subsection{Assessment Protocol}

Three baseline assessments with an interval of 2 weeks at the beginning of the training were made: one assessment in the middle of the training (MidTrain), one assessment at the end of the training (PostTrain), followed by another assessment one month after the training (End) (Figure 2). Multiple baseline assessments have commonly been used in other studies [52-55]. These baseline assessments were all done within a one-month period. Six clinical assessment sessions were done in total. Proprioception assessments were included in all clinical assessment sessions. An extra two sessions of the proprioceptive assessment only were performed following the 5 th clinical assessment session after the training period.

Two ipsilateral and contralateral arm matching tasks, each consisting of three movements (shoulder abduction, shoulder flexion, and elbow flexion), were used. Movements were performed at both 30 and 60 degree angles. For example, for the ipsilateral arm matching task (Figure 3), an experimenter positioned the affected arm of the participant at 30 degrees of shoulder abduction. The participant was requested to keep the arm in that position for $\sim 5 \mathrm{~s}$ before returning to a relaxed initial position. Then, after another $\sim 5 \mathrm{~s}$ delay, the participant moved the affected arm back to the remembered position. An experimenter measured this shoulder abduction angle manually using a goniometer. The same procedure was repeated for the 60 degree angle and for the other two movements. For the contralateral arm matching task (Figure 4), the procedure was the same as described above, except that the unaffected arm was moved first, and the patient had to match the angle with the affected arm.

A Fugl-Meyer assessment (FMA) [56], a Wolf motor function test (WMFT) [57], and a proprioception assessment [58] using angles measured with a goniometer were used for pre- and post-training evaluations. The FMA was applied for measuring UE and lower extremity (LE) motor and sensory impairment [56]. The FMA consists of 5 domains, which include motor, sensory, balance, range of motion, and joint pain domains. The assessment of UEs and LEs is part of the motor domain, which has a well-established reliability $[59,60]$. The validity of the FMA has been investigated with other scores, such as the functional independence measure, and an excellent correlation between the FMA and other scores was found [61]. The smallest real difference was considered to be $10 \%$ of the highest score [62]. The FMA is a three-point ordinal scale (0-2) and is used for the assessment of the motor and sensory FMA. Voluntary limb movement was assessed by the FMA motor assessment, which includes a UE subscale (33 items with a score range between 0 and 66) and an LE subscale (17 items with a score ranging between 0 and 34). The total motor FMA is 100 [56].

The WMFT is a time-based method for evaluating UE performance that consists of 15 tasks. The first 6 tasks consist of joint segment movements. The remaining tasks involve time-integrative functional movements [57]. The test-retest reliability of the WMFT is very high, and it has inter-rater reliability and internal consistency [63,64]. The WMFT has an excellent correlation with the action research arm test [63], suggesting it has good validity. In the literature, 1.5 to $2 \mathrm{~s}$ of change in the WMFT are considered to be clinically important [65].

For the proprioception assessment, a goniometer was used for pre- and post-training evaluations. Sabari et al. [66] have found a high intra-class correlation coefficient $>0.9$ intra-rater reliability for active and passive measurements at the same position, with comparable measurements between trial 1 and trial 2. 


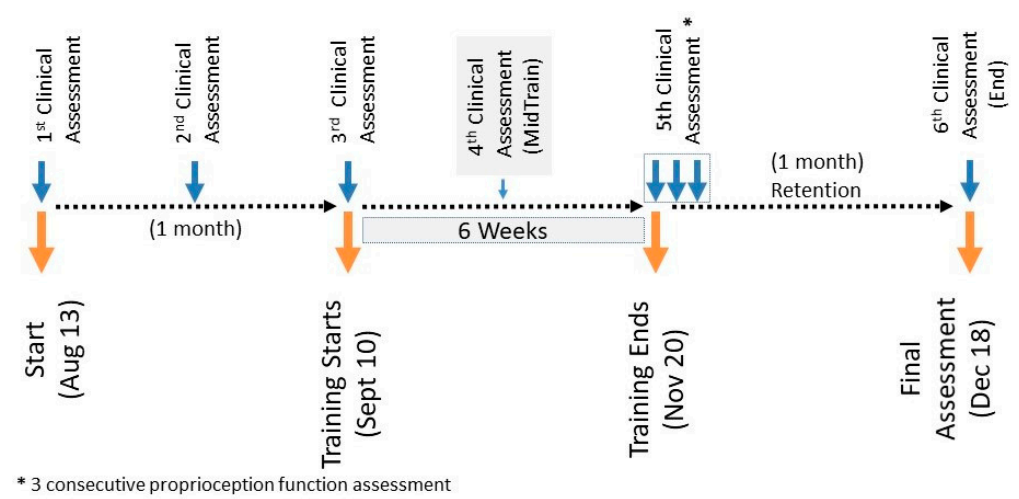

Figure 2. Study timeline.

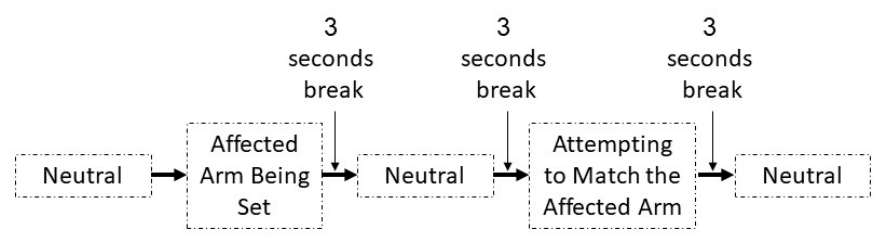

Figure 3. Steps in assessment of ipsilateral movements.

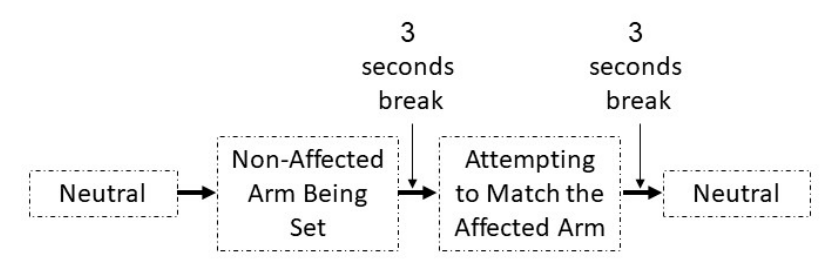

Figure 4. Contralateral movements assessment steps.

\section{System}

LabVIEW and Kinect were used for both training and assessment protocols. LabVIEW was used for the real-time data collection and analysis of the data received from Kinect.

\subsection{Receiver}

The receiver shown in Figure 5 consists of a power source, a power regulator and charger, an XBee receiver module, an optoisolator, and two bands (each connected to a vibrating motor).

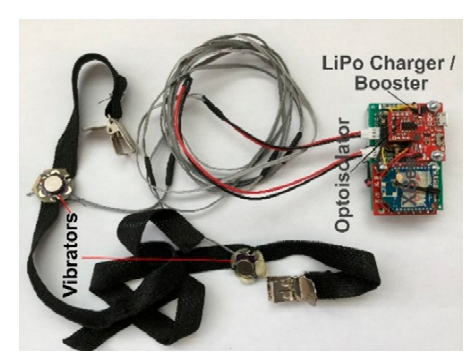

Figure 5. Prototype receiver.

\subsection{Transmitter}

A transmitter connected to a personal computer was used as an interface mean between the PC and the receiver. The circuitry and the prototyped transmitter are shown in Figure 6. The transmitter consists of an Arduino MEGA 2560, a logic level converter (BOB-12009), an XBee module, and two light emitting diodes (LEDs). The LEDs are used for representing the left and right arms/forearms for visual feedback purposes. 


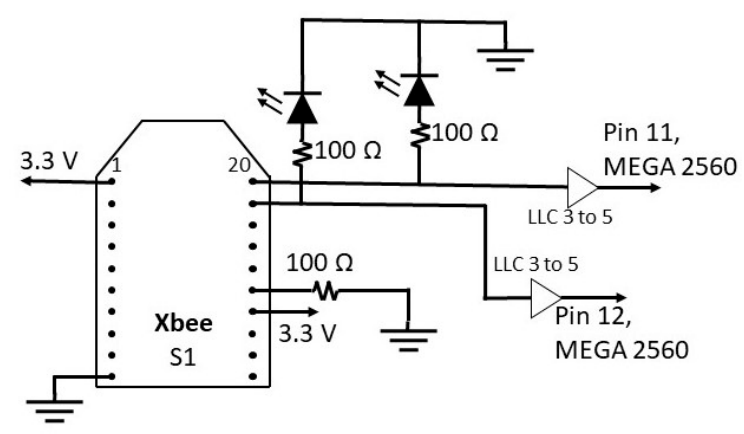

(A)

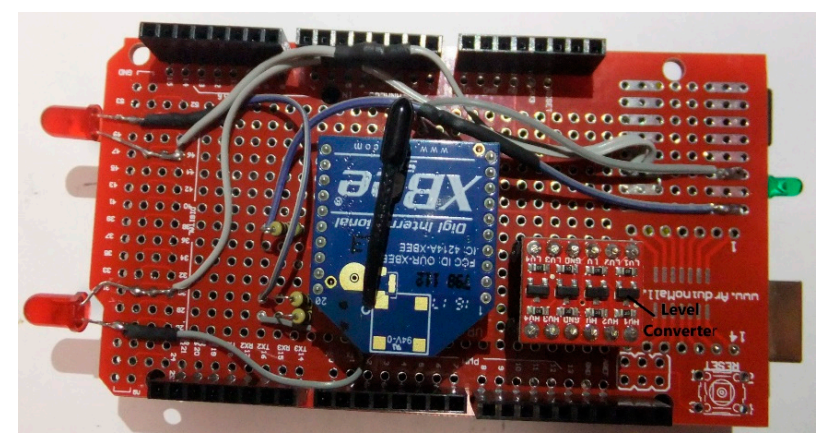

(B)

Figure 6. (A) Transmitter circuitry; (B) prototype transmitter. LLC 3 to 5 : logic level converter $3 \mathrm{~V}$ to $5 \mathrm{~V}$.

\subsection{Software and Hardware}

\subsubsection{Hardware}

Kinect was used to collect the user's body joint information. The joint positions are defined in the Kinect camera space, which consists of three single $x, y$, and $z$ positions. The origin of the camera space is the center of the depth or infrared sensor of the Kinect [67]. The joint position data are recorded by the Kinect accurately, while the joints are not occluded. However, this is not the case in terms of human movement $[68,69]$. The occlusion issue became even greater for some joints as the user carried out the movement while sitting.

XBee (XBee is the trademark of DIGI International, Inc.) was used for wireless communication between the receiver and transmitter. XBee is a module that allows for serial communication between devices over serial ports. "One-way of wireless communication" [70] was set up for the two XBee modules on the transmitter and receiver. XBee module settings were made by using XCTU (XCTU is a free multiplatform application provided by DIGI International, Inc.) and by using the following parameters (listed in Table 1).

Table 1. The parameters of the two XBee modules. DL sets the 16-bit address of the receiver XBee module; MY sets the 16-bit source address for the transmitter XBee module; D0, D1, and D2 are digital inputs or outputs; IR is the sample rate.

\begin{tabular}{ccc}
\hline Variables & Receiver & Transmitter \\
\hline ID & 3456 & 3456 \\
DL & 1 & 2 \\
MY & 2 & 1 \\
D0 & DO Low [4] & DI [3] \\
D1 & DO Low [4] & DI [3] \\
D2 & DO Low [4] & DI [3] \\
IR & 64 (for 100 ms) & 64 \\
IT & - & 1 \\
IA & 1 & - \\
\hline
\end{tabular}




\subsubsection{LabVIEW}

LabVIEW (LabVIEW is the trademark of National Instruments) was used as the interface between the Kinect and the computer using Haro3D (Haro3D is a product of HaroTek LLC), a library allowing users access to the functionalities of Kinect. In order to interface between LabVIEW and the transmitter, LINX was used. LINX is open source and provides an easy way for LabVIEW and Arduino to interact [71]. Upon installing the hardware abstraction layer on LabVIEW and then on Arduino by using LabVIEW (Tools $\rightarrow$ MakerHub $\rightarrow$ LINX $\rightarrow$ LINX $\rightarrow$ Firmware Wizard), a single interface between LabVIEW and Arduino MEGA 2560 was made, allowing for seamless communication. In other words, the virtual instruments of LINX were installed on the PC to communicate in a serial manner with the LINX firmware on the Arduino, which allowed for the manipulation of the Arduino output. Screenshots of the training and assessment protocols are shown in Figure 7.

Two tasks of "arm abduction" and "arm flexion" were carried out by the participant. The two joints wrist $(w)$ and shoulder (s) were chosen for upper limb movement comparison because both joints had minimal occlusion during the two movements. Both the $w$ and $s$ joints created two vectors, and the angle between the two line segments was defined as $\theta$ [72],

$$
\cos \theta=\frac{w^{T} s}{\|w \mid\| s \|}=\mathbf{u}^{T} \mathbf{v}
$$

where $\mathbf{u}$ and $\mathbf{v}$ are unit vectors and are expressed by the following formula:

$$
\mathbf{u}=\frac{1}{\|w\|} w \text { and } \mathbf{v}=\frac{1}{\|s\|} s .
$$

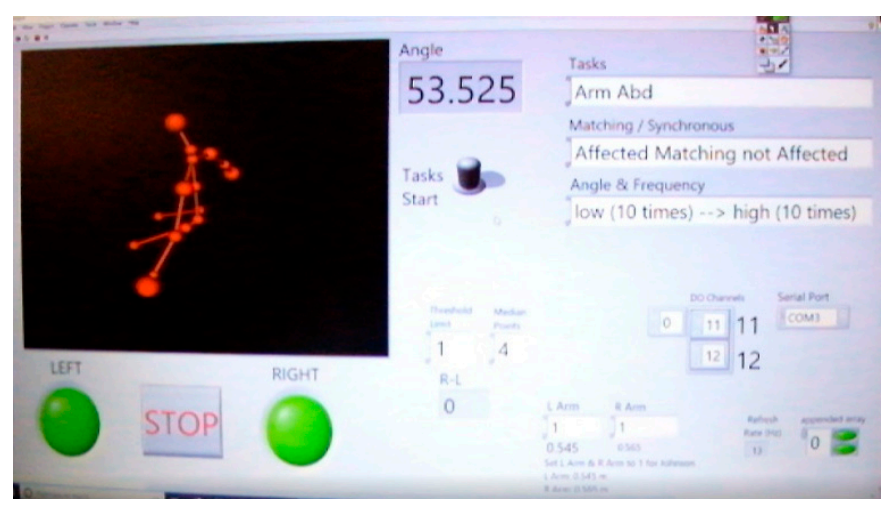

(A)

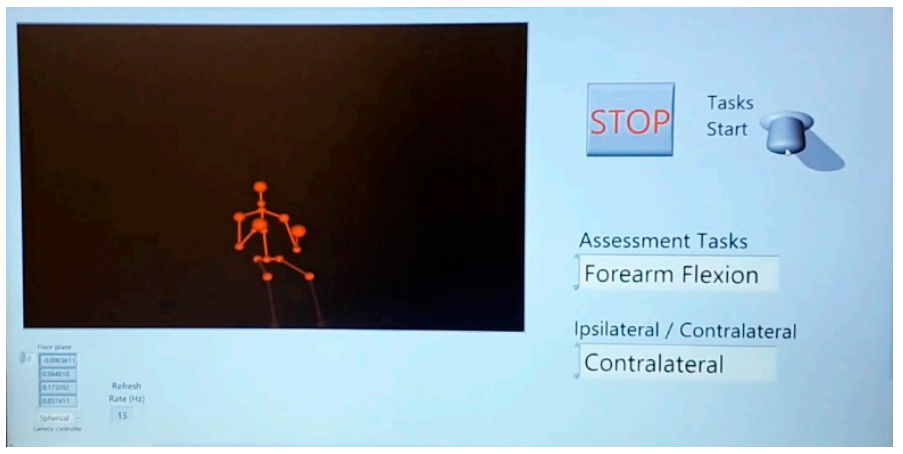

(B)

Figure 7. (A) LabVIEW training program; (B) LabVIEW assessment program. 
The Euclidean distance between joints $w$ and $s$ was found as 72

$$
\|w-s\|=\sqrt{\left(x_{w}-x_{s}\right)^{2}+\left(y_{w}-y_{s}\right)^{2}+\left(z_{w}-z_{s}\right)^{2}},
$$

where $w$ and $s$ stand for wrist and shoulder joint position data, respectively.

The distance between $w$ and $s$ was used for movement comparisons because it required a minimum amount of computations. Considering the real-time processing of the Kinect camera information, methods with a lower computational cost were preferable.

\section{Results}

The data collected from a left hemiplegic participant based on a timeline study (shown in Figure 2) were analyzed to assess motor function (FMA and WMFT) and proprioception function (ipsilateral and contralateral arm matching angular assessments). Three assessment plots are shown in Figures 8-10. The three baseline clinical assessment values used as baselines are drawn as boxplots in the subplots of the three plots of Figures 8-10. The fourth, fifth, and sixth assessments are specified as MidTrain, PostTrain, and Retention. The scores of these three assessments are shown as black-filled dots. Motor function and proprioception assessment plots are discussed below.
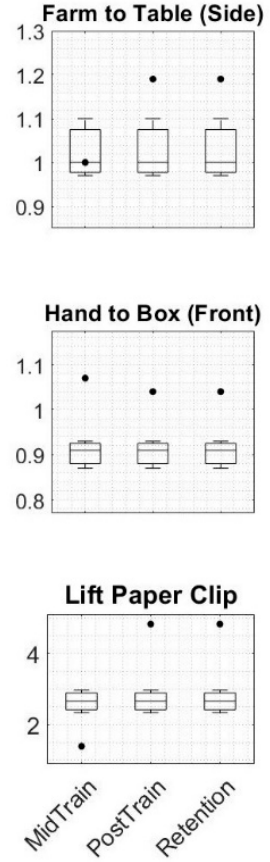
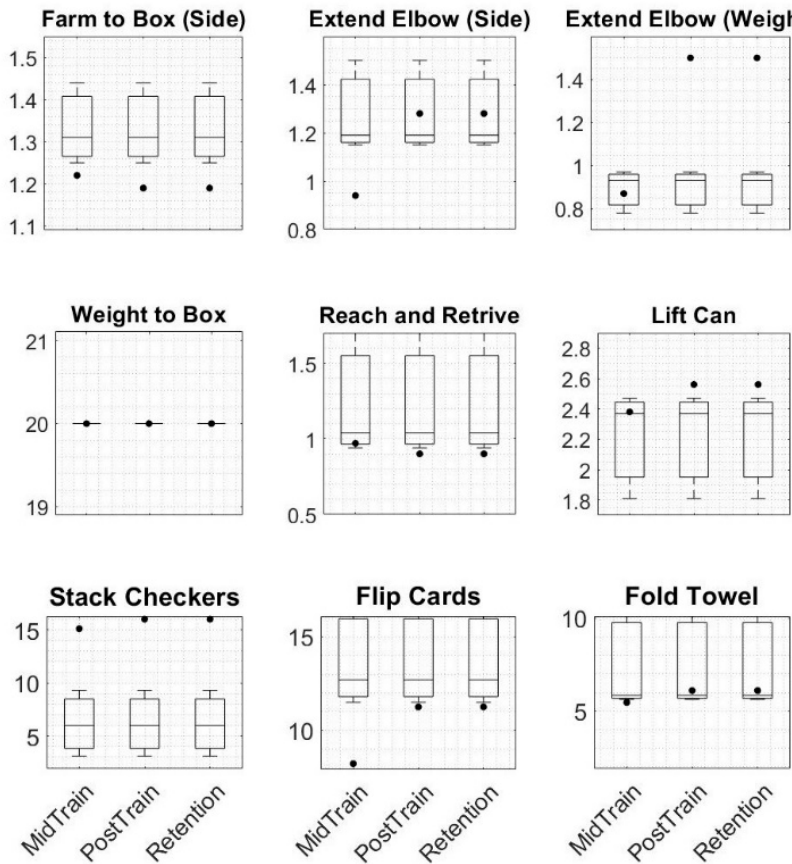
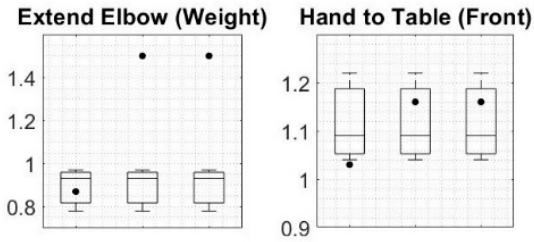

0.9
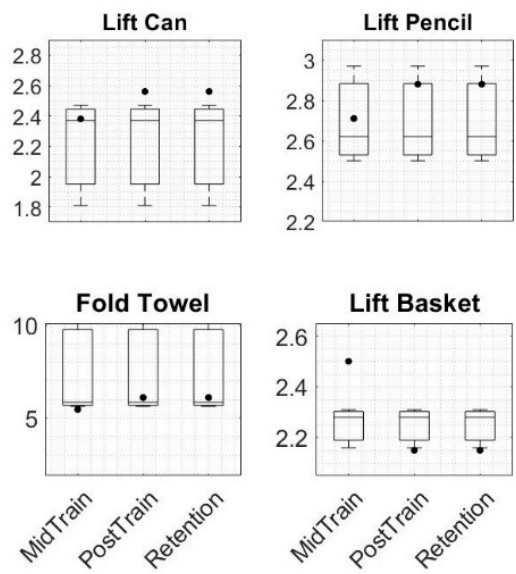

Figure 8. The boxplots for the Wolf motor function test (WMFT) are shown. The three baselines are plotted as boxplots in subplots. The scores for mid training, post training, and retention (one month after six weeks of training) are shown as black-filled dots and are respectively named MidTrain, PostTrain, and Retention. The $y$ axis does not have any unit. The total scores of the four assessments were 65, 65, 74, and 66, respectively. Abbreviations: Farm, forearm. 


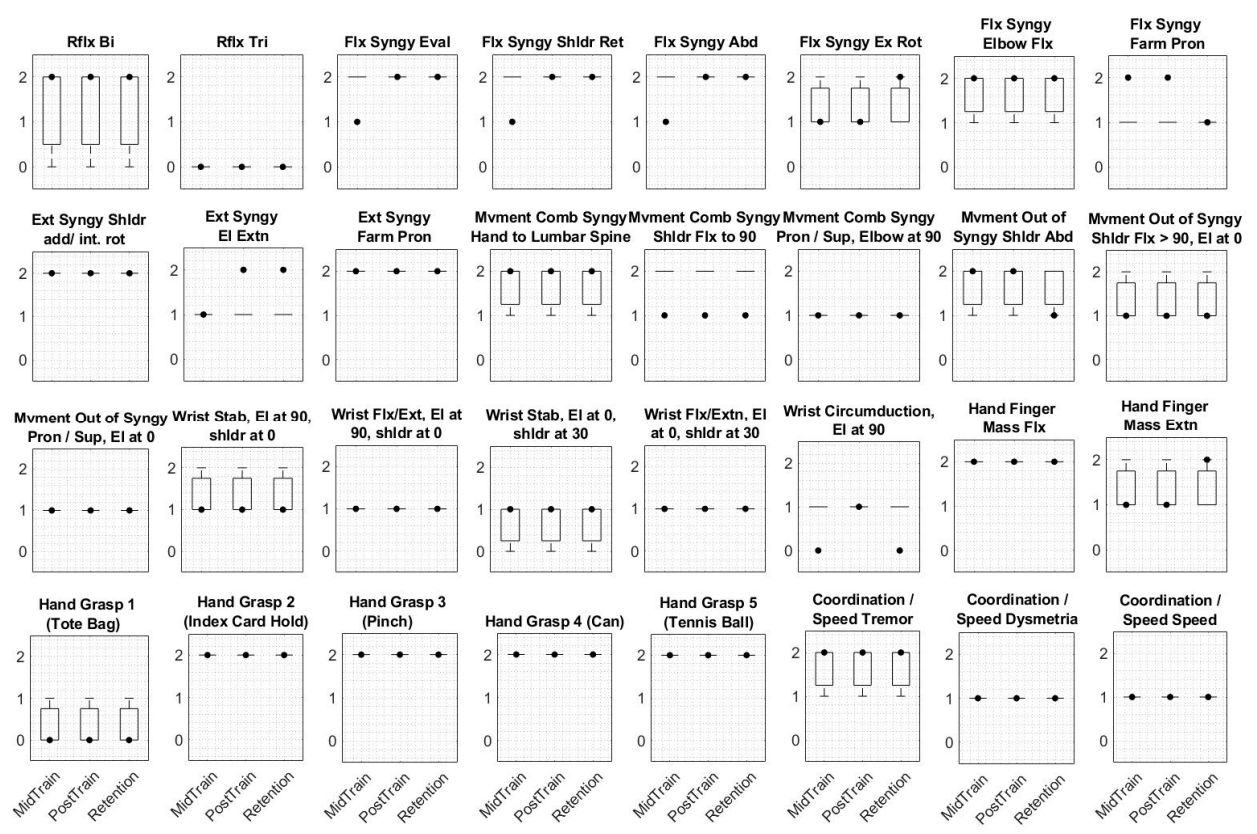

Figure 9. The boxplots for the Fugl-Meyer assessment (FMA) are shown. The three baselines are plotted as boxplots in subplots. The scores for mid training, post training, and retention (one month after six weeks of training) are shown as black-filled dots and are respectively named MidTrain, PostTrain, and Retention. The total scores of the four assessments were 45.33,42, 47, and 46, respectively. The $y$ axis does not have any unit. Abbreviations: Rflx, reflex; Bi: biceps; Tri, triceps; Flx, flexor; Syngy, synergy; Eval, evaluation; Shldr, shoulder; Ret, retraction; Abd, abduction; Ex, external; Rot, rotation; Pron, pronation; Ext, extensor; add, adduction; Int, internal; rot, rotation; El, elbow; Farm, forearm; Mvment, movement; Comb, combining; Sup, supination; Extn, extension; Stab, stability; 90, 90 degrees; 0, 0 degrees.
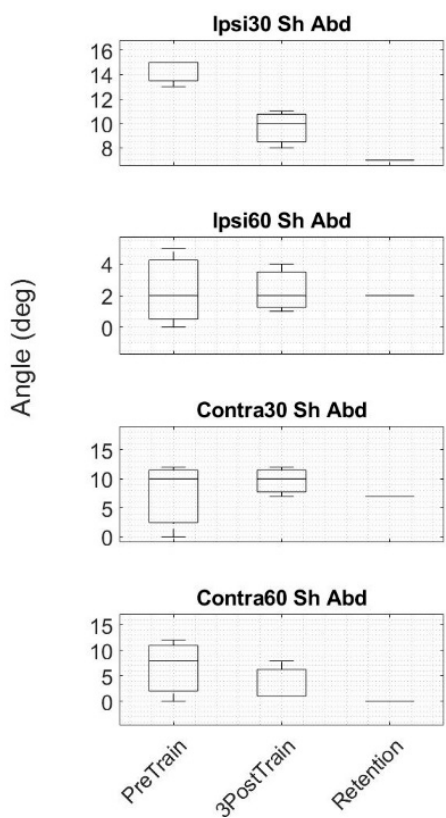
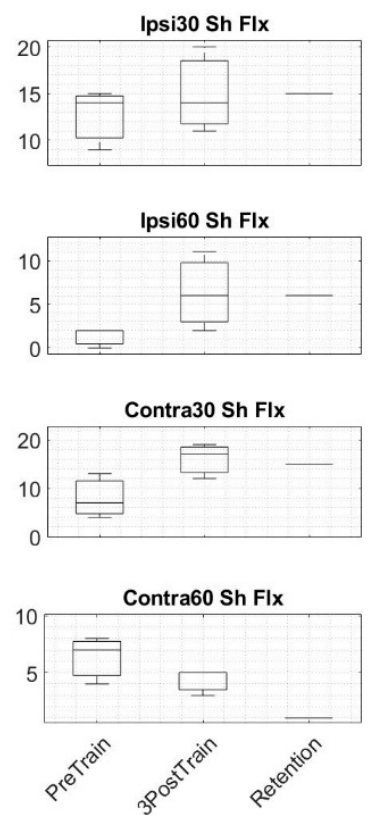

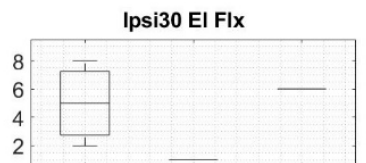

Ipsi60 El Flx
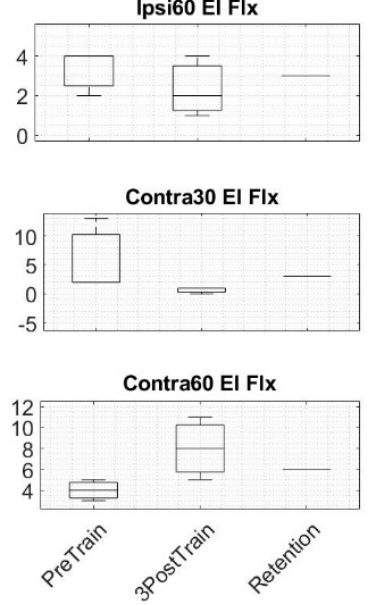

Figure 10. The boxplots for goniometer angle assessments of 12 tasks are shown. PreTrain: boxplot of the three baseline assessments before training starts; 3PostTrain: boxplot of the three consecutive assessments after training ends; Retention: assessment value after one month of training. Abbreviations: Ipsi, ipsilateral; Contra, contralateral; Sh, shoulder; Abd, abduction; Flx, flexion; El, elbow; 30, 30 degrees; 60, 60 degrees. 


\subsection{Motor Function}

The scores of the PostTrain and Retention assessments for the WMFT (Figure 8) showed deterioration for nine of the items compared to the baseline scores. Four items showed improvement, and two showed no differences.

Except for seven tasks that showed improvement on the Retention assessment for the FMA (Figure 9), the rest of the tasks showed almost no difference compared to the mean values of the boxplots.

\subsection{Proprioception}

Measurements for each angle (30 degrees and 60 degrees) for shoulder abduction, shoulder flexion, and elbow flexion for both "ipsilateral arm matching" and "contralateral arm matching" were collected using a goniometer. The results are shown in Figure 10. The Retention assessment scores compared to PreTrain assessment values showed improvement in four conditions (30 degrees of ipsilateral shoulder abduction, 30 degrees of contralateral shoulder abduction, 60 degrees of contralateral shoulder abduction, and 30 degrees of contralateral elbow flexion). Three pre- and post-training values were considered as two groups of independent variables. A Kruskal-Wallis H test was used for the two groups' statistical analysis. At a 1\% significance level, no significant difference was found between the two groups.

\section{Discussion}

The objective of this study was to design and develop a bimanual rehabilitation/training system combined with tactile feedback and Kinect for stroke survivors to verify the feasibility of our methodology. The participant was able to perform two types of trainings protocols (ipsilateral and contralateral arm matching) successfully by getting haptic feedback. The results showed improvement in some conditions after six weeks of training. Even for some tasks with no or less than median improvement (60 degrees of ipsilateral shoulder abduction (Ipsi60 Sh Abd) or 30 degrees of contralateral shoulder abduction (Contra30 Sh Abd), for example), the percentile angular errors were reduced, which is encouraging. Compared to 30 degrees of angular motion, 60 degrees of angular motion showed less improvement in both the median and the percentiles. Three reasons are proposed for this observation. First, the stiffness and soreness in the muscles of the shoulder affected the larger abduction and flexion movements. This was a fact that the participant himself confirmed. Second, as the traveling distance to a target increased, larger errors were produced [73]. Third, the weakness of the deltoid muscles as the result of paralysis influenced the range of motion.

During the proprioception assessments, the participant's arm was passively positioned by an experimenter, and as a result, the time between positioning differed. The different time length between two positionings yielded different perceptions in the participant, which is called a Tau effect [74]. Herrnstadt et al. [18] have suggested that the need to memorize the location of an arm is eliminated, but the Tau effect might have been triggered, influencing the participant's judgement. Additionally, passive arm positioning was shown to result in larger errors compared to active arm positioning [75].

Bimanual systems have been successfully applied in many clinical studies [9-12], and it has been shown that UE bimanual training is more beneficial to stroke patients compared to unilateral UE training [12]. Consistent with the meta-analysis of Chen et al. [12] on the comparison of bilateral and unilateral upper limb training in people with stroke, our results showed improvement of the stroke participant in terms of FMA total score. Although we saw improvement in the FMA total score of the participant, this may not have necessarily yielded significant improvement in functional improvement. The bimanual training concept has been combined with robotics [76-81] and with auditory cueing [82-86] in several studies for the rehabilitation of the UEs of stroke patients. Providing the user with physical feedback has been used in previous studies. For instance, force feedback [49] and active assisted and active resist modes for increasing cerebral activation levels [87] are examples of such feedback that has been used in studies. For our study, we embedded tactile 
feedback in our novel training protocol, which required active engagement of the user during the training and proved to be effective for motor improvement [88]. Including haptic feedback in our study was useful for the blindfolded participant of our study during training. He had a little trouble in knowing the location of his affected left arm. The haptic feedback assisted him to match his affected arm to the position of his unaffected arm.

The application of Kinect in our combined bimanual training with haptic feedback was novel and allowed us to verify the potential of Kinect for a self-training system for stroke survivors.

As Hackshaw [89] has suggested, conducting a well-designed small study is appropriate as long as its results are interpreted carefully, because small studies suffer from reliable estimation. In our study, we recruited one suitable stroke participant from our available list of stroke individuals. While this study helped us to confirm the feasibility of our training system, it did not firmly confirm the clinical results. A larger study will help in developing better training and assessment protocols to suit a wider range of stroke patients with mild to moderate paralysis. It will also enhance our estimation of the clinical results. For example, if we look at the FMA results of the participant, we noticed a modest improvement compared to other FMA results in the literature (e.g., Reference [90]), which made any conclusion on the significance of the clinical results difficult. The second limitation of our study was the length of the training. The participant could successfully finish six weeks of training, but we could not confirm the long-term use of the training system and the effect of tactile feedback. These two points should be addressed in future studies.

\section{Conclusions}

Rehabilitation and training are the key to recovery after stroke. Considering increasing amounts of healthcare costs, patient self-training/rehabilitation is primordial for a sustainable economy. In order to investigate the potential of an affordable and easy-to-use system for rehabilitation/training, we designed and developed a protocol and training system for stroke patients. We tested the system on a left hemiplegic participant, who was able to successfully complete six weeks of training, which showed promise in terms of system and protocol design. We also performed several functional assessments before and after the training. The proprioception assessments showed improvement in some tasks, indicating a positive effect of our protocol in rehabilitating stroke survivors.

Author Contributions: Conceptualization, A.O.; Methodology, A.O., E.E.A., C.M.; Formal Analysis and Validation, A.O., E.E.A., X.Z., H.M., C.M.; Investigation, A.O., X.Z., H.M., C.M.; Resources, C.M.; Data Curation, A.O., X.Z., H.M., C.M.; Writing Original Draft, A.O., Review and Editing, A.O., E.E.A., C.W.L., X.Z., C.M.; Supervision, C.M.; Administration, C.W.L., C.M.; Funding, C.W.L., C.M.

Funding: This research was supported by the Canadian Institutes of Health Research (CIHR) with a grant number of 353444 .

Acknowledgments: The authors wish to thank the participant in the study.

Conflicts of Interest: The authors declare no conflict of interest.

\section{References}

1. Hossmann, K.A. Pathophysiology and Therapy of Experimental Stroke. Cell. Mol. Neurobiol. 2006, 26, 1057-1083. [CrossRef] [PubMed]

2. Warlow, C.P.; Van Gijn, J.; Dennis, M.S.; Wardlaw, J.M.; Bamford, J.M.; Hankey, G.J.; Sandercock, P.A.; Rinkel, G.; Langhorne, P.; Sudlow, C.; et al. Stroke Practical Management, 3rd ed.; Oxford Blackwell Publishing: Oxford, UK, 2008.

3. Bonita, R.; Mendis, S.; Truelsen, T.; Bogousslavsky, J.; Toole, J.; Yatsu, F. The global stroke initiative. Lancet Neurol. 2004, 3, 391-393. [CrossRef]

4. $\quad$ Cramer, S.C.; Nelles, G.; Benson, R.R.; Kaplan, J.D.; Parker, R.A.; Kwong, K.K.; Kennedy, D.N.; Finklestein, S.P.; Rosen, B.R. A functional mri study of subjects recovered from hemiparetic stroke. Stroke 1997, 28, 2518-2527. [CrossRef] [PubMed] 
5. Bleyenheuft, Y.; Gordon, A.M. Precision grip in congenital and acquired hemiparesis: Similarities in impairments and implications for neurorehabilitation. Front. Hum. Neurosci. 2014, 8, 459. [CrossRef] [PubMed]

6. Buschfort, R.; Brocke, J.; Heß, A.; Werner, C.; Waldner, A.; Stefan, H. Arm studio to intensify upper limb rehabilitation after stroke: Concept, acceptance, utilization and preliminary clinical results. J. Rehabil. Med. 2010, 42, 310-314. [CrossRef] [PubMed]

7. Gowland, C.; deBruin, H.; Basmajian, J.; Plews, N.; Nurcea, I. Agonist and Antagonist Activity During Voluntary Upper Limb Movement in Patients with Stroke. Phys. Ther. 1992, 72, 624-633. [CrossRef] [PubMed]

8. Gresham, G.E.; Fitzpatrick, T.E.; Wolf, P.A.; McNamara, P.M.; Kannel, W.B.; Dawber, T.R. Residual Disability in Survivors of Stroke-The Framingham Study. N. Engl. J. Med. 1975, 293, 954-956. [CrossRef] [PubMed]

9. Latimer, C.P.; Keeling, J.; Lin, B.; Henderson, M.; Hale, L.A. The impact of bilateral therapy on upper limb function after chronic stroke: A systematic review. Disabil. Rehabil. 2010, 32, 1221-1231. [CrossRef]

10. Van Delden, A.E.Q.; Peper, C.E.; Beek, P.J.; Kwakkel, G. Unilateral versus bilateral upper limb exercise therapy after stroke: A systematic review. J. Rehabil. Med. 2012, 44, 106-117. [CrossRef]

11. Stewart, K.C.; Cauraugh, J.H.; Summers, J.J. Bilateral movement training and stroke rehabilitation: A systematic review and meta-analysis. J. Neurol. Sci. 2006, 244, 89-95. [CrossRef]

12. Chen, P.M.; Kwong, P.W.H.; Lai, C.K.Y.; Ng, S.S.M. Comparison of bilateral and unilateral upper limb training in people with stroke: A systematic review and meta-analysis. PLoS ONE 2019, 14, e0216357. [CrossRef] [PubMed]

13. Coldberg, G. Supplementary motor area structure and function: Review and hypothesies. Behav. Brain Sci. 1985, 8, 567-616. [CrossRef]

14. Swinnen, S.P.; Wenderoth, N. Two hands, one brain: Cognitive neuroscience of bimanual skill. Trends Cogn. Sci. 2004, 8, 18-25. [CrossRef] [PubMed]

15. Waller, S.M.C.; Whitall, J. Bilateral arm training: Why and who benefits? NeuroRehabilitation 2008, $23,29-41$.

16. Lee, M.J.; Lee, J.H.; Koo, H.M.; Lee, S.M. Effectiveness of Bilateral Arm Training for Improving Extremity Function and Activities of Daily Living Performance in Hemiplegic Patients. J. Stroke Cerebrovasc. Dis. 2017, 26, 1020-1025. [CrossRef] [PubMed]

17. Cauraugh, J.H.; Lodha, N.; Naik, S.K.; Summers, J.J. Bilateral movement training and stroke motor recovery progress: A structured review and meta-analysis. Hum. Mov. Sci. 2010, 29, 853-870. [CrossRef] [PubMed]

18. Herrnstadt, G.; Alavi, N.; Randhawa, B.K.; Boyd, L.A.; Menon, C. Bimanual elbow robotic orthoses: Preliminary investigations on an impairment force-feedback rehabilitation method. Front. Hum. Neurosci. 2015, 9, 1-14. [CrossRef] [PubMed]

19. Lum, P.S.; Burgar, C.G.; Van der Loos, M.; Shor, P.C. MIME robotic device for upper-limb neurorehabilitation in subacute stroke subjects: A follow-up study. J. Rehabil. Res. Dev. 2006, 43, 631. [CrossRef]

20. Van Delden, A.L.E.Q.; Peper, C.L.E.; Kwakkel, G.; Beek, P.J. A Systematic Review of Bilateral Upper Limb Training Devices for Post Stroke Rehabilitation. Stroke Res. Treat. 2012, 2012, 1-12. [CrossRef]

21. Richards, L.G.; Senesac, C.R.; Davis, S.B.; Woodbury, M.L.; Nadeau, S.E. Bilateral arm training with rhythmic auditory cueing in chronic stroke: Not always efficacious. Neurorehabilit. Neural Repair 2008, 22, 180-184. [CrossRef]

22. Jones, L.A.; Sarter, N.B. Tactile Displays: Guidance for Their Design and Application. Hum. Factors 2008, 50, 90-111. [CrossRef] [PubMed]

23. Bark, K.; Wheeler, J.; Shull, P.B.; Savall, J.; Cutkosky, M. Rotational skin stretch feedback: A wearable haptic display for motion. IEEE Trans. Haptics 2010, 3, 166-176. [CrossRef] [PubMed]

24. Shull, P.B.; Damian, D.D. Haptic wearables as sensory replacement, sensory augmentation and trainerA review. J. Neuroeng. Rehabil. 2015, 12, 1-13. [CrossRef] [PubMed]

25. Sigrist, R.; Rauter, G.; Riener, R.; Wolf, P. Augmented visual, auditory, haptic, and multimodal feedback in motor learning: A review. Psychon. Bull. Rev. 2013, 20, 21-53. [CrossRef] [PubMed]

26. Stepp, C.E.; Matsuoka, Y. Object manipulation improvements due to single session training outweigh the differences among stimulation sites during vibrotactile feedback. IEEE Trans. Neural Syst. Rehabil. Eng. 2011, 19, 677-685. [CrossRef] [PubMed]

27. Schiefer, M.A.; Graczyk, E.L.; Sidik, S.M.; Dustin, D.W.T. Artificial tactile and proprioceptive feedback improves performance and confidence on object identification tasks. PLoS ONE 2018, 13, e0207659. [CrossRef] 
28. Held, J.P.; Klaassen, B.; van Beijnum, B.J.F.; Luft, A.R.; Veltink, P.H. Usability Evaluation of a VibroTactile Feedback System in Stroke Subjects. Bioeng. Biotechnol. 2017, 4, 98. [CrossRef]

29. Diego-Mas, J.A.; Alcaide-Marzal, J. Using Kinect sensor in observational methods for assessing postures at work. Appl. Ergon. 2014, 45, 976-985. [CrossRef]

30. Xu, X.; Robertson, M.; Chen, K.B.; Lin, J.H.; McGorry, R.W. Using the Microsoft KinectTM to assess 3-D shoulder kinematics during computer use. Appl. Ergon. 2017, 65, 418-423. [CrossRef]

31. Galna, B.; Barry, G.; Jackson, D.; Mhiripiri, D.; Olivier, P.; Rochester, L. Accuracy of the Microsoft Kinect sensor for measuring movement in people with Parkinson's disease. Gait Posture 2014, 39, 1062-1068. [CrossRef]

32. Webster, D.; Celik, O. Systematic review of Kinect applications in elderly care and stroke rehabilitation. J. Neuroeng. Rehabil. 2014, 11, 1-24. [CrossRef] [PubMed]

33. Kim, W.S.; Cho, S.; Park, S.H.; Lee, J.Y.; Kwon, S.Y.; Paik, N.J. A low cost kinect-based virtual rehabilitation system for inpatient rehabilitation of the upper limb in patients with subacute stroke: A randomized, double-blind, sham-controlled pilot trial. Medicine 2018, 97, 1-8. [CrossRef] [PubMed]

34. Da Gama, A.; Chaves, T.; Figueiredo, L.; Teichrieb, V. Poster: Improving motor rehabilitation process through a natural interaction based system using kinect sensor. In Proceedings of the 2012 IEEE Symposium on 3D User Interfaces (3DUI), Costa Mesa, CA, USA, 4-5 March 2012; pp. 145-146.

35. Pastor, I.; Hayes, H.A.; Bamberg, S.J. A feasibility study of an upper limb rehabilitation system using kinect and computer games. In Proceedings of the 2012 Annual International Conference of the Engineering inMedicine and Biology Society (EMBC), San Diego, CA, USA, 28 August-1 September 2012; pp. 1286-1289.

36. Yeh, S.C.; Hwang, W.Y.; Huang, T.C.; Liu, W.K.; Chen, Y.T.; Hung, Y.P. A study for the application of body sensing in assisted rehabilitation training. In Proceedings of the 2012 International Symposium on Computer, Consumer and Control (IS3C), Taichung, Taiwan, 4-6 June 2012; pp. 922-925.

37. Shiratuddin, M.F.; Hajnal, A.; Farkas, A.; Wong, K.W.; Legradi, G. A proposed framework for an interactive visuotactile $3 \mathrm{D}$ virtual environment system for visuomotor rehabilitation of stroke patients. In Proceedings of the 2012 International Conference on Computer \& Information Science (ICCIS), Kuala Lumpur, Malaysia, 12-14 June 2012; pp. 1052-1057.

38. Frisoli, A.; Loconsole, C.; Leonardis, D.; Banno, F.; Barsotti, M.; Chisari, C.; Bergamasco, M. A new gaze-BCI-driven control of an upper limb exoskeleton for rehabilitation in real-world tasks. IEEE Trans. Syst. Man Cybern. C 2012, 42, 1169-1179. [CrossRef]

39. Tuthill, C.T.; Azim, E. Proprioception. Curr. Biol. 2018, 28, R187-R207. [CrossRef] [PubMed]

40. Kiper, P.; Baba, A.; Agostini, M.; Turolla, A. Proprioceptive Based Training for stroke recovery. Proposal of new treatment modality for rehabilitation of upper limb in neurological diseases. Arch. Physiother. 2015, 5, 1-6. [CrossRef] [PubMed]

41. Chae, S.H.; Kim, Y.L.; Lee, S.M. Effects of phase proprioceptive training on balance in patients with chronic stroke. J. Phys. Ther. Sci. 2017, 29, 839-844. [CrossRef] [PubMed]

42. Clark, N.C.; Röijezon, U.; Treleaven, J. Proprioception in musculoskeletal rehabilitation. Part 2: Clinical assessment and intervention. Man. Ther. 2015, 20, 378-387. [CrossRef]

43. Rand, D. Proprioception deficits in chronic stroke-Upper extremity function and daily living. PLoS ONE 2018, 13, e0195043. [CrossRef]

44. Smith, D.L.; Akhtar, A.J.; Garraway, W.M. Proprioception and spatial neglect after stroke. Age Ageing 1983, 12, 63-69. [CrossRef]

45. El-Gohary, T.M.; Khaled, O.A.; Ibrahim, S.R.; Alshenqiti, A.M.; Ibrahim, M.I. Effect of proprioception cross training on repositioning accuracy and balance among healthy individuals. J. Phys. Ther. Sci. 2016, 28, 3178-3182. [CrossRef]

46. Carroll, T.J.; Herbert, R.D.; Munn, J.; Lee, M.; Gandevia, S.C. Contralateral effects of unilateral strength training: Evidence and possible mechanisms. J. Appl. Physiol. 2006, 101, 1514-1522. [CrossRef] [PubMed]

47. Lee, M.; Gandevia, S.C.; Carroll, T.J. Unilateral strength training increases voluntary activation of the opposite untrained limb. Clin. Neurophysiol. 2009, 120, 802-808. [CrossRef] [PubMed]

48. Muaidi, Q.I.; Nicholson, L.L.; Refshauge, K.M.; Adams, R.D.; Roe, J.P. Effect of anterior cruciate ligament injury and reconstruction on proprioceptive acuity of knee rotation in the transverse plane. Am. J. Sports Med. 2009, 37, 1618-1626. [CrossRef] [PubMed] 
49. Johnson, J.J.; Van der Loos, M.; Brugar, C.G.; Shor, P.; Leifer, L.J. Experimental Results Using Force-Feedback Cueing in Robot-Assisted Stroke Therapy. IEEE Trans. Neural Syst. Rehabil. Eng. 2005, 13, 335-348. [CrossRef] [PubMed]

50. Morimoto, T.; Schreiner, A.S.; Asano, H. Caregiver burden and health-related quality of life among Japanese stroke caregivers. Age Ageing 2003, 32, 218-223. [CrossRef] [PubMed]

51. Tombaugh, T.N.; McIntyre, N.J. The Mini-Mental State Examination: A Comprehensive Review. J. Am. Geriatr. Soc. 1992, 40, 922-935. [CrossRef] [PubMed]

52. Zhang, X.; Elnady, A.M.; Randhawa, B.K.; Boyd, L.A.; Menon, C. Combining Mental Training and Physical Training with Goal-Oriented Protocols in Stroke Rehabilitation: A Feasibility Case Study. Front. Hum. Neurosci. 2018, 12, 125. [CrossRef] [PubMed]

53. Lobo, M.A.; Moeyaert, M.; Cunha, A.B.; Babik, I. Single-case design, analysis, and quality assessment for intervention research. J. Neurol. Phys. Ther. JNPT 2017, 41, 187. [CrossRef]

54. Kratochwill, T.R.; Hitchcock, J.; Horner, H.; Levin, J.R.; Odom, S.L.; Rindskopf, D.M.; Shadish, W.R. Single-Case Designs Technical Documentation. Available online: https://eric.ed.gov/?id=ED510743 (accessed on 28 July 2019).

55. Dijkerman, H.C.; Webeling, M.; ter Wal, J.M.; Groet, E.; van Zanvoort, M.J. A long-lasting improvement of somatosensory function after prism adaptation, a case study. Neuropsychologia 2004, 42, 1697-1702. [CrossRef] [PubMed]

56. Sullivan, K.J.; Tilson, J.K.; Cen, S.Y.; Rose, D.K.; Hershberg, J.; Correa, A.; Gallichio, J.; McLeod, M.; Moore, C.; $\mathrm{Wu}$, S.S.; et al. Fugl-Meyer Assessment of Sensorimotor Function After Stroke Standardized Training Procedure for Clinical Practice and Clinical Trials. Stroke 2011, 42, 427-432. [CrossRef] [PubMed]

57. Wolf, S.L.; Catlin, P.A.; Ellis, M.; Archer, A.L.; Morgan, B.; Piacentino, A. Assessing Wolf Motor Function Test as Outcome Measure for Research in Patients After Stroke. Stroke 2001, 32, 1635-1639. [CrossRef] [PubMed]

58. Hillier, S.; Immink, M.; Thewlis, D. Assessing Proprioception: A Systematic Review of Possibilities. Neurorehabil. Neural Repair. 2015, 29, 933-949. [CrossRef] [PubMed]

59. Duncan, P.W.; Propst, M.; Nelson, S.G. Reliability of the Fugl-Meyer assessment of sensorimotor recovery following cerebrovascular accident. Phys. Ther. 1983, 63, 1606-1610. [CrossRef] [PubMed]

60. Gladstone, D.J.; Danells, C.J.; Black, S.E. The Fugl-Meyer assessment of motor recovery after stroke: A critical review of its measurement properties. Neurorehabil. Neural Repair 2002, 6, 232-240. [CrossRef] [PubMed]

61. Rabadi, M.H.; Rabadi, F.M. Comparison of the action research arm test and the Fugl-Meyer assessment as measures of upper-extremity motor weakness after stroke. Arch. Phys. Med. Rehabil. 2006, 87, 962-966. [CrossRef] [PubMed]

62. Hsueh, I.P.; Hsu, M.J.; Sheu, C.F.; Lee, S.; Hsieh, C.L.; Lin, J.H. Psychometric comparisons of 2 versions of the Fugl-Meyer Motor Scale and 2 versions of the Stroke Rehabilitation Assessment of Movement. Neurorehabilit. Neural Repair 2008, 22, 737-744. [CrossRef] [PubMed]

63. Nijland, R.; van Wegen, E.; Verbunt, J.; van Wijk, R.; van Kordelaar, J.; Kwakkel, G. A comparison of two validated tests for upper limb function after stroke: The Wolf Motor Function Test and the Action Research Arm Test. J. Rehabil. Med. 2010, 42, 694-696. [PubMed]

64. Whitall, J.; Savin, D.; Harris-Love, M.; Waller, S. Psychometric properties of a modified wolf motor function test for people with mild and moderate upper extremity hemiparesis. Arch. Phys. Med. Rehabil. 2006, 82, 750-755. [CrossRef]

65. Lin, K.C.; Hsieh, Y.W.; Wu, C.Y.; Chen, C.L.; Jang, Y.; Liu, J.S. Minimal detectable change and clinically important difference of the Wolf Motor Function Test in stroke patients. Neurorehabilit. Neural Repair 2009, 23, 429-434. [CrossRef]

66. Sabari, J.S.; Maltzev, I.; Lubarsky, D.; Liszkay, E.; Homel, P. Goniometric Assessment of Shoulder Range of Motion: Comparison of Testing in Supine and Sitting Positions. Rev. Bras. Med. Esporte. 2012, 18, $38-41$. [CrossRef]

67. Haro3D. 3D Visualization Library for LabVIEW, User guide version 2.2; Harotek: Keller, TX, USA, 2018. Available online: http://www.harotek.com/products (accessed on 20 April 2018).

68. Huang, H.Y.; Chang, S.H. A Skeleton-occluded Repair Method from Kinect. In Proceedings of the IEEE 2014 International Symposium on Computer, Consumer and Control, Taichung, Taiwan, 10-12 June 2014.

69. Moon, S.; Park, Y.; Ko, D.W.; Suh, H. Multiple Kinect Sensor Fusion for Human Skeleton Tracking Using Kalman Filtering. Int. J. Adv. Robot. Syst. 2016, 13, 65. [CrossRef] 
70. Titus, J. The Hands-On XBee Lab Manual, 1st ed.; Elsevier: Amsterdam, The Netherlands, 2012.

71. LabVIEW Makerhub. Available online: https://www.labviewmakerhub.com/doku.php?id=libraries:linx:start (accessed on 20 April 2018).

72. Leon, S.J. Linear Algebra with Applications, 9th ed.; Pearson Education Limited: London, UK, 2015.

73. Lönn, J.; Crenshaw, A.G.; Djupsjöbacka, M.; Pedersen, J.; Johansson, H. Position Sense Testing: Influence of Starting Position and Type of Displacement. Arch. Phys. Med. Rehabil. 2015, 81, 592-596. [CrossRef]

74. Helson, H.; King, S.M. The tau effect: An example of pxychological relativity. J. Exp. Psychol. 1931, 14, 202-217. [CrossRef]

75. Fuentes, C.T.; Bastian, A.J. Where is your arm? Variations in proprioception across space and tasks. J. Neurophysiol. 2010, 103, 164-171. [CrossRef]

76. Lin, C.-H.; Chou, L.-W.; Luo, H.-J.; Tsai, P.-Y.; Lieu, F.-K.; Chiang, S.-L.; Sung, W.H. Effects of computer-aided Interlimb force coupling training on paretic hand and arm motor control following chronic stroke: A randomized controlled trial. PLoS ONE 2015, 10, e0131048. [CrossRef] [PubMed]

77. Kim, H.; Miller, L.M.; Fedulow, I.; Simkins, M.; Abrams, G.M.; Byl, N.; Rosen, J. Kinematic data analysis for post-stroke patients following bilateral versus unilateral rehabilitation with an upper limb wearable robotic system. IEEE Trans. Neural Syst. Rehabil. Eng. 2013, 21, 153-164. [CrossRef]

78. Liao, W.W.; Wu, C.Y.; Hsieh, Y.W.; Lin, K.C.; Chang, W.Y. Effects of robot-assisted upper limb rehabilitation on daily function and real-world arm activity in patients with chronic stroke: A randomized controlled trial. Clin. Rehabil. 2012, 26, 111-120. [CrossRef]

79. Wu, C.Y.; Yang, C.L.; Lin, K.C.; Wu, L.L. Unilateral versus bilateral robot-assisted rehabilitation on arm-trunk control and functions post stroke: A randomized controlled trial. J. Neuroeng. Rehabil. 2013, 10, 35. [CrossRef]

80. Hsieh, Y.W.; Wu, C.Y.; Wang, W.E.; Lin, K.C.; Chang, K.C.; Chen, C.C.; Liu, C.T. Bilateral robotic priming before task-oriented approach in subacute stroke rehabilitation: A pilot randomized controlled trial. Clin. Rehabil. 2017, 31, 225-233. [CrossRef]

81. Yang, C.L.; Lin, K.C.; Chen, H.C.; Wu, C.Y.; Chen, C.L. Pilot comparative study of unilateral and bilateral robot-assisted training on upper-extremity performance in patients with stroke. Am. J. Occup. Ther. 2012, 66, 198-206. [CrossRef]

82. Luft, A.R.; McCombe-Waller, S.; Whitall, J.; Forrester, L.W.; Macko, R.; Sorkin, J.D.; Schulz, J.B.; Goldberg, A.P.; Hanley, D.F. Repetitive bilateral arm training and motor cortex activation in chronic stroke: A randomized controlled trial. JAMA 2004, 292, 1853-1861. [CrossRef] [PubMed]

83. Whitall, J.; Waller, S.M.; Sorkin, J.D.; Forrester, L.W.; Macko, R.F.; Hanley, D.F.; Goldberg, A.P.; Luft, A. Bilateral and unilateral arm training improve motor function through differing neuroplastic mechanisms: A single-blinded randomized controlled trial. Neurorehabilit. Neural Repair 2011, 25, 118-129. [CrossRef] [PubMed]

84. Van Delden, A.L.; Peper, C.L.; Nienhuys, K.N.; Zijp, N.I.; Beek, P.J.; Kwakkel, G. Unilateral versus bilateral upper limb training after stroke: The Upper Limb Training After Stroke clinical trial. Stroke 2013, 44, 2613-2616. [CrossRef] [PubMed]

85. Waller, S.M.; Liu, W.; Whitall, J. Temporal and spatial control following bilateral versus unilateral training. Hum. Mov. Sci. 2008, 27, 749-758. [CrossRef] [PubMed]

86. Shahine, E.M.; Shafshak, T.S. The effect of repetitive bilateral arm training with rhythmic auditory cueing on motor performance and central motor changes in patients with chronic stroke. Egypt. Rheumatol. Rehabil. 2014, 41, 8. [CrossRef]

87. Li, C.; Inoue, Y.; Liu, T.; Sun, L. Validation of bimanual-coordinated training supported by a new upper-limb rehabilitation robot: A near-infrared spectroscopy study. Disabil. Rehabil. Assist. Technol. 2013, 8, 38-48. [CrossRef] [PubMed]

88. Basteris, A.; Nijenhuis, S.M.; Stienen, A.H.; Buurke, J.H.; Prange, G.B.; Amirabdollahian, F. Training modalities in robot-mediated upper limb rehabilitation in stroke: A frame work for classification based on a systematic review. J. Neuroeng. Rehabil. 2014, 11, 111. [CrossRef] 
89. Hackshaw, A. Small studies: Strengths and limitations. Eur. Respir. J. 2008, 32, 1141-1143. [CrossRef]

90. Wu, C.Y.; Hsieh, Y.W.; Lin, K.C.; Chuang, L.L.; Chang, Y.F.; Liu, H.L.; Chen, C.L.; Lin, K.H.; Wai, Y.Y. Brain Reorganization after Bilateral Arm Training and Distributed Constraint-induced Therapy in Stroke Patients: A Preliminary Functional Magnetic Resonance Imaging Study. Chang. Gung Med. J. 2010, 33, 628-637. 